\title{
In Situ Light Dosimetry During Photodynamic Therapy of Barrett's Esophagus With 5-Aminolevulinic Acid
}

\author{
Robert L.P. van Veen, ${ }^{1}$ Maurice C.G. Aalders, $\mathrm{PhD},{ }^{4}$ Kasper L. Pasma, $\mathrm{PhD},{ }^{2}$ Peter D. Siersema, MD, PhD, ${ }^{3}$ \\ Jelle Haringsma, $\mathrm{MD}^{3}{ }^{3}$ Wim van de Vrie, $\mathrm{MD}^{3,5}$ Edward E.E. Gabeler, ${ }^{3 D}{ }^{5}{ }^{\text {Dominic J. Robinson, PhD, }}{ }^{1}$ \\ and Henricus J.C.M. Sterenborg, $\mathrm{PhD}^{1 *}$ \\ ${ }^{1}$ Photodynamic Therapy and Optical Spectroscopy Program, Erasmus MC, Rotterdam, The Netherlands \\ ${ }^{2}$ Department of Radiation Oncology, Erasmus MC, Rotterdam, The Netherlands \\ ${ }^{3}$ Department of Gastroenterology \& Hepatology, Erasmus MC, Rotterdam, The Netherlands \\ ${ }^{4}$ Laser Center, Academic Medical Centre, Amsterdam, The Netherlands \\ ${ }^{5}$ Department of Surgery, Erasmus MC, Rotterdam, The Netherlands
}

Background and Objectives: Previous studies with PhotoDynamic Therapy (PDT) in bladder and bronchi have shown that due to scattering and reflection, the actually delivered fluence rate on the surface in a hollow organ can be significantly higher than expected. In this pilot study, we investigated the differences between the primary calculated and the actual measured fluence rate during PDT of Barrett's Esophagus (BE) using 23 independent clinical measurements in 15 patients.

Study Design/Materials and Methods: A KTP-dye module laser at $630 \mathrm{~nm}$ was used as light source. Light delivery was performed using a cylindrical light diffuser inserted in the center of an inflatable transparent balloon with a length corresponding to the length of the Barrett's epithelium. The total light output power of the cylindrical diffuser was calibrated using an integrating sphere to deliver a primary fluence rate of $100 \mathrm{~mW} \mathrm{~cm}^{-2}$. Two fiber-optic pseudo sphere isotropic detectors were placed on the balloon and were used to measure fluence rate at the surface of the esophageal wall during PDT.

Results and Conclusions: The actual fluence rate measured was 1.5-3.9 times higher than the primary fluence rate for $630 \mathrm{~nm}$. In general, the fluence rate amplification factor decreased with increasing redness of the tissue and was less for shorter diffusers. Fluence rate variations in time were observed which coincided with patients coughing, movement, and esophageal spasms. These factors combined with inter patient variability of the fluence rate measured appears to justify the routine application of this technique in PDT of BE. Lasers Surg. Med. 31:299-304, 2002.

(c) 2002 Wiley-Liss, Inc.

Key words: fluence; fluence rate; isotropic detectors; back scattering

\section{INTRODUCTION}

Barrett's Esophagus (BE) is a premalignant condition for which superficial ablation might be used to reduce the risk of malignant degeneration. The current therapy for high- grade dysplasia is still esophagectomy but this is comprised by a short-term morbidity of $30 \%$. PhotoDynamic Therapy (PDT) using 5 aminolevulinic acid (ALA) as a pro-drug for the production of PpIX acting as photosensitiser has found world wide interest as a potential tool for treating early stage BE. ALA-PDT eliminates the epithelium of BE selectively without damaging the entire wall of the esophagus due to selective localisation of PpIX in the oesophageal mucosa rather than in the underlying stroma [1-3]. PDT for the superficial ablation of Barrett's mucosa might be superior to the existing therapies due to less morbidity and mortality compared to surgery.

For PDT, the time interval between photosensitiser administration and illumination-scheme is important. More recently de Bruijn et al. [4] suggested a two-fold illumination scheme for which a dramatic increase in tumour volume doubling time was demonstrated. Furthermore, light fluence rate and the fluence (also referred to as light dose) delivered to the tissue were demonstrated to strongly influence the severity of PDT-induced tissue damage [5]. In previous oesophageal PDT studies [1,6-11], light dosimetry was defined as either an estimation of the primary fluence rate (based on the total output power of a cylindrical diffuser divided by the area to be treated) or merely the light output power per length of diffuser. This approach assumes a radially emitting cylindrical diffuser and does not account for tissue light backscattering and reflection. In previous experiences with PDT in bladder and bronchi [12,13] we have shown that the actually delivered fluence rate in a hollow organ can be significantly higher than the primary fluence rate, due to back-scattering of light from the tissue and reflection at the tissue surface boundaries. The tissue optical and geometrical properties are different in each

\footnotetext{
*Correspondence to: H.J.C.M. Sterenborg, PhD, Photodynamic Therapy and Optical Spectroscopy Programme, Department of Radiation Oncology, Daniel den Hoed Cancer Center, Erasmus MC, P.O. Box 5201, 3008 AE Rotterdam, The Netherlands.

E-mail: sterenborg@kfih.azr.nl

Accepted 29 August 2002

Published online in Wiley InterScience

(www.interscience.wiley.com).

DOI 10.1002/lsm.10129
} 
patient, consequently the actual fluence rate will vary, and thus may lead to either under-treatment or over-treatment. Measuring the actual fluence rate at the oesophageal surface can be expected to improve the correlation between treatment parameters and treatment effect and may ensure a safer illumination.

To evaluate the practical necessity of in vivo dosimetry, we developed a balloon based light delivery system with integrated light detection system and performed measurements of the actual fluence rate during PDT in the esophagus of 15 patients. The purpose of the dosimetry study was: (a) to investigate the difference between the actual and primary fluence rate, (b) to evaluate the necessity of performing such measurements on a routine basis, and (c) to investigate possible differences in fluence rate during the second session resulting from PDT effects of the first session of a two-fold illumination scheme. The dosimetric study described in this paper was performed during a clinical pilot experiment investigating a double illumination scheme for PDT of BE. The treatment was still based on the estimation of the primary fluence rate, hence measuring the true fluence rate was of no consequence for the treatment.

In this paper, we present the results of in situ light dosimetry during PDT of 15 patients resulting in 23 independent measurements. From these results, we have derived fluence rate amplification factors for all illuminations. All parameters that may contribute to this factor will be discussed.

\section{MATERIALS AND METHODS}

\section{Light Dosimetry}

A schematic representation of the inflatable balloon light delivery/dosimetry is depicted in Figure 1.

The aim was to deliver a primary fluence rate $\left(\phi_{\text {primary }}\right)$ of $100 \mathrm{~mW} \mathrm{~cm}^{-2}$ to the esophageal surface based on a first order approximation of the primary fluence rate in absence of tissue.

$$
\phi_{\text {primary }}=\frac{P_{\text {out }}}{\pi \cdot D_{\text {ball }} \cdot L_{\text {dif }}}
$$

where $D_{\text {ball }}$ is the balloon diameter [cm], $P_{\text {out }}$ the cylindrical diffuser total output power $[\mathrm{mW}]$, and $L_{\text {dif }}$ length

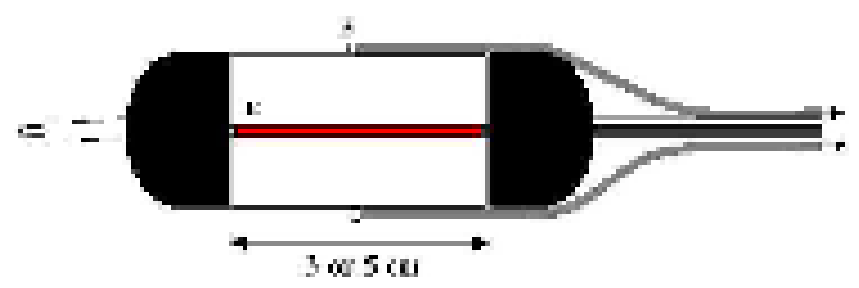

Fig. 1. Light applicator with incorporated dosimetry for PDT of the Barrett's esophagus (BE) consisting of an inflated windowed transparent balloon with (a) two counter-opposite isotropic detectors tape in the middle, and (b) a channel for the cylindrical diffuser. (Color figure can be viewed in the online issue, which is available at www.interscience.wiley.com) [cm] of the diffuser corresponding to the Barretts epithelium length [14]. In this approach, the cylindrical diffuser is assumed to behave as a radially emitting line source with the output homogeneously distributed over the cylinder surface. Prior to BE PDT the total output power of the cylindrical diffuser measured in an integrating sphere (Grasbery Optronics S370, Zevenaar, TeLintelo systems, The Netherlands) and was set to a value yielding a calculated fluence rate of $100 \mathrm{~mW} \mathrm{~cm} \mathrm{~cm}^{-2}$ for the 3 and $5 \mathrm{~cm}$ cylindrical diffusers, respectively, i.e., $3.9 \mathrm{~W}$ for a $5 \mathrm{~cm}$ diffuser in a $2.5 \mathrm{~cm}$ balloon diameter.

The fluence rate at the surface of the applicator was measured using a fiber ( $400 \mu \mathrm{m}$ core diameter) with an out site diameter of $1 \mathrm{~mm}$ pseudo sphere isotropic detector at the distal end (Cardiofocus formerly known as Rare Earth Medical, West Yarmouth MA), thus measuring the sum of the primary incident light from the cylindrical diffuser and the tissue remitted and reflected light. The response of the detectors was isotropic within 5\%. A detailed description on isotropic detectors is given by van Staveren et al. [15]. The isotropic probes were connected to an electronic device that enables real time fluence (rate) measurements. The electronic device was connected to a PC for storage and processing of the measured data. Calibration of the detectors was performed in a built-in integrating sphere that provides a well-defined diffuse calibration field. The dosimetry software applied a factor to correct for the difference in response of the detectors when measurements are performed at the air/tissue surface resulting from mismatch in refractive index [16]. Light delivery was performed using an inflatable transparent windowed balloon (Wizzard X-cell, Wilson-Cook Medical, Inc., WinstonSalem, NC) The interior of the black non-transparent capped portions on both sides is coated with a silver-grey reflective material [17]. Prior to PDT, two probes were taped (transparent Scotch Magic tape, 3M) to the middle of the balloon opposite to each other. During PDT, the two isotropic detectors recorded the fluence rate $\left(\phi_{1,2}\right)$ every second at two position at the BE surface. Balloon diameter was $2.5 \mathrm{~cm}$ with a transparent window length of either 3 or $5 \mathrm{~cm}$. A KTP/532-dye laser module at $630 \mathrm{~nm}$ (Laserscope, San Jose, CA) was used for light delivery. A cylindrical diffuser (400 $\mu \mathrm{m}$ fibre core diameter) (CeramOptec, $\mathrm{GmbH}$, Bonn, Germany) was aligned in the balloon prior to the procedure. The length of the balloon and cylindrical diffuser was chosen to be either 3 or $5 \mathrm{~cm}$, corresponding to the estimated length of the Barrett's epithelium. This resulted in two different illumination geometries. Prior to PDT, a white light endoscopic surveillance was performed and the guide-wire was positioned. The deflated balloon was positioned over the guide-wire and then inflated to a pressure of approximately $60-\mathrm{mm} \mathrm{Hg}$ and its position endoscopically checked. After the guide-wire was removed the cylindrical diffuser was inserted. The output power of the cylindrical diffuser was checked prior and post PDT.

\section{Data Processing}

The fluence rate was measured every second at two locations at the BE surface $\phi_{1}(t), \phi_{2}(t)$. 
The mean of the actual measured fluence rate per illumination session was calculated according to Equation 2.

$$
\phi_{\text {actual }}=\frac{1}{T} \int_{0}^{T} \frac{\phi_{1}(t)+\phi_{2}(t)}{2} \mathrm{~d} t
$$

where $T$ stands for the total illumination time in seconds. For each patient the fluence rate amplification factor $F$ is determined which is the ratio of the actual fluence rate $\phi_{\text {actual }}$ and the first order approximation of the primary fluence rate in absence of tissue according to Equation 3 [13].

$$
F_{i, j}=\frac{\phi_{\text {actual }}}{\phi_{\text {primary }}}
$$

For each patient $(i)$ the fluence rate amplification factor for first and second illumination $(j)$ were calculated. To investigate possible acute tissue response as result of the first illumination we have determined if there was a significant difference between $F_{i, 1}$ and $F_{i, 2}$ could be observed.

\section{PATIENTS}

In this prospective study, 15 patients with $\mathrm{BE}$ with estimated lengths varying from 2 to $5 \mathrm{~cm}$, with low and high-grade dysplasia were randomly subdivided in various illumination schemes. After oral administration of $60 \mathrm{mg} /$ kg ALA (Sigma-Aldrich Chemie BV. Zwijdrecht, Netherlands). PDT illumination schemes were: single illumination of $100 \mathrm{~J} \mathrm{~cm}^{-2} 4$ hours after ALA administration, or fractionated: either two times $100 \mathrm{~J} \mathrm{~cm}^{-2}$ (4 and 5 hours after ALA administration) or $20-100 \mathrm{~J} \mathrm{~cm}^{-2}$ ( 1 and 4 hours after ALA administration). The patients were sedated with an intravenously injection of $5-10 \mathrm{mg}$ midazolam Dormicum $^{\mathbb{B}}$, Roche Nederland B.V., Mijndrecht, The Netherlands).

\section{RESULTS}

In Figures 2 and 3 examples of in situ fluence rate measurements during PDT are shown. Figure 2 illustrates the fluence rate versus treatment time of a patient receiving a two-fold illumination with a intended primary fluence of 20 and $100 \mathrm{~J} \mathrm{~cm}^{-2}$, and $\phi_{\text {primary }} 100 \mathrm{~mW} \mathrm{~cm}^{-2}$ at $630 \mathrm{~nm}$. The average fluence rates during the first illumination period were $\phi_{1}=152 \pm 7 \mathrm{~mW} \mathrm{~cm}^{-2}$ and $\phi_{2}=162 \pm 5 \mathrm{~mW}$ $\mathrm{cm}^{-2}$ and showed relatively small variations for the two detector positions 1 and 2, respectively. The average fluence rate delivered to the tissue during the second illumination period was $\phi_{1}=168 \pm 22 \mathrm{~mW} \mathrm{~cm}^{-2}$ and $\phi_{2}=173 \pm 25$ $\mathrm{mW} \mathrm{cm}{ }^{-2}$ for the two detector positions, respectively. The fluence rate amplification factors as calculated with Equation 3 were $F_{i, 1}=1.6$ and $F_{i, 2}=1.7$ for the first and second illumination period, respectively.

Figure 3 shows the fluence rate versus treatment time of a patient receiving a two-fold illumination with a intended primary fluence of two times $100 \mathrm{~J} \mathrm{~cm}^{-2}$, $\phi_{\text {primary }}=100 \mathrm{~mW}$ $\mathrm{cm}^{-2}$ at $630 \mathrm{~nm}$. Large variations in fluence rate were measured. During the first illumination period, the dif-

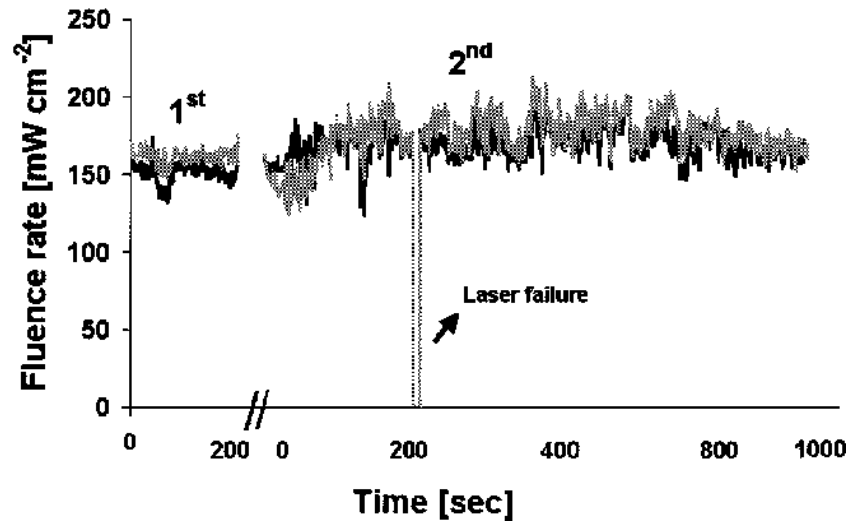

Fig. 2. Fluence rate $\left[\mathrm{mW} \mathrm{cm}^{-2}\right]$ measured in time [second] during a two-fold PDT illumination $630 \mathrm{~nm}$ of the BE at two opposite positions ( $\phi_{1}, \phi_{2}$ at 1 sample per second). The fluence rates amplification factors were 1.6 and 1.7 during the first and second illumination respectively (according to Eq. 3). The desired calculated fluence was 20 and $100 \mathrm{~J} \mathrm{~cm}^{-2}$ using a fluence rate of $100 \mathrm{~mW} \mathrm{~cm}^{-2}$ (Eq. 1). Diffuser length $L_{\text {dif }}=3 \mathrm{~cm}$ and the balloon diameter $D_{\text {ball }}=2.5 \mathrm{~cm}$.

ference in actual fluence rate between both locations was a factor 1.6. The fluence rate amplification factors $F_{i, 1}$ and $F_{i, 2}$ were 3.3 and 2.7 for the first and second illumination period.

Coughing, and spasm of the esophagus corresponded with large variations in fluence rate as recorded during PDT, smaller variations of the signal are possibly due to motion artefacts from respiration and heartbeat. The largest variation coincided with patient movement. Smaller variations were recorded when the patient was calm after additional administration of midazolam.

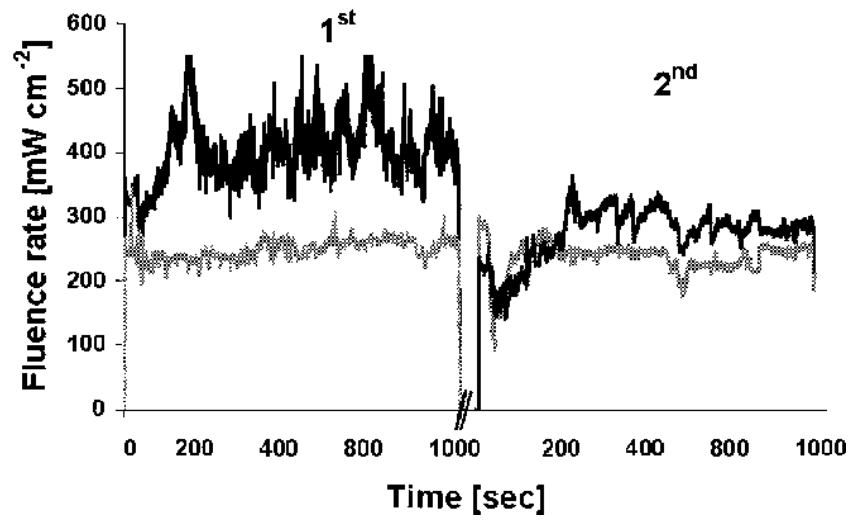

Fig. 3. Fluence rate $\left[\mathrm{mW} \mathrm{cm}^{-2}\right]$ measured in time [second] during a two-fold PDT illumination $630 \mathrm{~nm}$ of the BE at two opposite positions ( $\phi_{1}, \phi_{2}$ at 1 sample per second). The fluence rate amplication factors (Eq. 3) were on average 3.3 and 2.7 during the first and second illumination respectively. The desired calculated fluence was $2 \times 100 \mathrm{~J} \mathrm{~cm}^{-2}$ using a fluence rate of $100 \mathrm{~mW} \mathrm{~cm}^{-2}$ (Eq. 1). Diffuser length $L_{\mathrm{dif}}=5 \mathrm{~cm}$ and the balloon diameter $D_{\text {ball }}=2.5 \mathrm{~cm}$. 
In eight patients, the fluence rate amplification factor $F$ was determined twice during a two-fold illumination. If the treatment would influence the optical properties of the tissue enough, then the second $F$ would always differ in the same manner for all patients. A paired $t$-test was used to test the hypothesis $F_{i, 1}=F_{i, 2}$, the $t$ value was found to be -0.13321 at 12 degrees of freedom, i.e., that at any reasonable significance level there is no significant difference between $F_{i, 1}, F_{i, 2}$ during the first and the second illumination in a single patient.

Figure 4 summarises the fluence rate amplification $F_{i, 1}$ and $F_{i, 2}$ factors during PDT for all patients as a function of illumination geometries, i.e., ratio of diffuser length and balloon diameter $\left(L_{\text {dif }} / D_{\text {ball }}\right)$. A Students $t$-test revealed significant difference $(P<0.005)$ between both geometries. The average fluence rate amplification factors $F$ for both geometries were 1.8 (SD 0.5) and 2.8 (SD 0.5), respectively.

\section{CONCLUSION}

As seen in Figure 3, the fluence rate can either increase at one measurement position while remaining stable at the other, or increase or decrease at both positions simultaneously. This gives a clue to the nature of these variations. When the patient coughs or an esophageal spasm occurs, muscles contract around the balloon. This squeezes blood out of the tissue, resulting in an increase in fluence rate build-up factor on both measurement locations simultaneously. The single sided variations should have a more local origin. Normal squamous epithelium often has a whitish colour whereas Barrett's columnar epithelium is

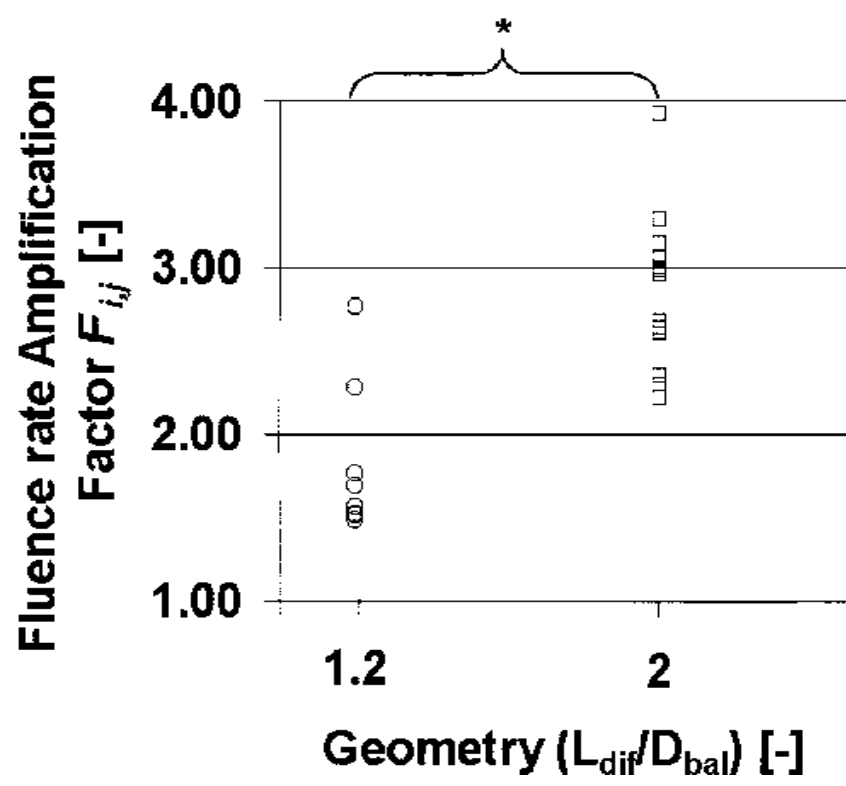

Fig. 4. The fluence rate amplification factor $F_{i, j}$ (measured, i.e., scattered plus nonscattered fluence rate, divided by the calculated fluence rate) as a functions of the illumination geometries used. Each point represents a patient $F$ during the first or second illumination. more reddish-pink. Due to movement of the balloon, the position of the isotropic detectors at the mucosa surface will vary slightly. Thus when a detector is positioned in the vicinity of a clear junction between squamous and Barrett's epithelium, local fluence rate variations may also occur. Although the fluence rate amplification is not a local effect, but mainly influenced by the optical properties of the whole esophageal wall, local colour variations will influence the actual fluence rate measured at that certain location.

The cylindrical diffuser was not fixed in the middle of the balloon but usually slightly bent, one probe was always located closer to the diffuser, thus measuring the highest primary fluence rate at the balloon surface. The large difference in fluence rate between both positions during the first illumination as seen in Figure 3, may be attributed to an eccentric position of the cylindrical diffuser resulting from the patients spasms. Murrer et al. [18] investigated the influence of off-axis positioned cylindrical diffuser using Monte Carlo simulations in a trachea model and showed that central off-axis deviations may result in serious overdosing at the surface closest to the cylindrical diffuser. As seen in Figure 4, the fluence rate amplification factor depends on the illumination geometry. In case of small diffuser lengths, relative more light is scattering isotropically into the absorbing part of the balloon (Fig. 1). Due to this, the contribution of backscattered light to the measured fluence rate at the tissue surface will be smaller. This may explain the difference in fluence rate build-up factor between the two diffuser lengths. Panjehpour et al. [19,20] also measured the fluence (rate) in a normal canine esophagus model using isotropic detectors. They found relatively small variations of approximately $\pm 12 \%$ between the $(n=8)$ different animals. In that study the detectors were placed on three healthy tissue positions. From their data we have calculated a fluence rate amplification factor $F=0.64$ according to Equation 3 . In a similar animal study by Overholt et al. [21], using a $180^{\circ}$ windowed balloon (black coating over half the balloon window length) the measured fluence rate was shown to be less than with a $360^{\circ}$ balloon. From their data we calculated an $F$ of 0.43 , which is much lower than what was measured in the present study. The explanation for this apparent discrepancy may be due to the illumination geometry and less backscattering from the balloon surface. Murrer et al. [18] described the cylindrical diffuser as the superposition of a series of isotropic point sources. This approach predicts a smaller fluence rate amplification factor for shorter diffusers due to axial loss of light. In addition, the effect of multiple reflections on the fluence rate amplification factor, not included in this analysis, will increase the losses for shorter diffuser lengths.

Van den Bergh et al. [22] highlighted the importance of standardising illumination devices and treatment procedures. They concluded that the comparison of the results between centres performing PDT in the esophagus would be much improved if procedures and light delivery devices we optimised and that this may also lead to improved treatment outcomes. Treatment outcome is also influenced by photosensitiser selectivity. Insufficient selectivity may 
result in stenosis or fistulas could be compensated to a certain extent with accurate light dosimetry as opposed by Bays et al. [23]. Furthermore, they had measured the fluence rate distribution as a function of penetration depth in ex-vivo human esophagus tissue. From their data we have derived a fluence rate amplification factor at the oesaphagus mucosa surface of approximately 2 and 1.7 for 630 and $514 \mathrm{~nm}$, respectively. van den Boogert et al. (1998) [24] found similar results for in vivo measurement in an animal study; $F=2.4$ and $F=1.3$ for $\lambda=633 \mathrm{~nm}$ and $\lambda=532 \mathrm{~nm}$, respectively and found less epithelial damage at $523 \mathrm{~nm}$ which was attributed to differences in optical properties, e.g., strong blood absorption and higher scattering resulting in a lower $F$. PDT of the BE using $514 \mathrm{~nm}$ (unpublished data) resulted in fluence rate amplification factors ranging from 0.8 to 1.8. The use of small isotropic detectors placed at the mucosa tissue surface will not result in under dosing underneath the detector surface as light will be scattered around the isotropic detector [25].

In this study large variations were seen between the calculated and measured fluence rate, furthermore differences by a factor 2 to 3 in fluence rate were measured between the two opposite detectors, these variations may seriously alter the clinical response. For photosensitisers such as ALA induced PpIX risk of over-treatment is not very large, due to photobleaching. Insufficient treatment, however, may increase local recurrences, which has been observed frequently. With other photosensitisers like $m$-THPC [26], or Photofrin [27] over-treatment may result in excessive PDT damage.

A large variability of the fluence rate was observed during PDT. Firstly, a major cause of variation was patient movement, coughing, and esophageal spasm. Secondly, the fluence rate amplification factor varied in the range of 1.5-3.9 for 15 patients and appeared to depend on the redness of the BE. Thirdly, eccentric positioning of the cylindrical diffuser resulted in significant difference in fluence rate delivered to opposite sites of the $\mathrm{BE}$ esophagus. Finally, the actual fluence rate delivered to the mucosal surface depended on the illumination geometry. All these factors appear to justify the routine application of in vivo dosimetry in PDT of BE especially for potent photosenstisers such as $\mathrm{HpD}$ or $m$-THPC. No significant differences in fluence rate amplification were measured between the first and second illumination during a two-fold PDT.

\section{REFERENCES}

1. Barr H, Shepherd NA, Dix A, Roberts DJ, Tan WC, Krasner N. Eradication of high-grade dysplasia in columnar-lined (Barrett's) esophagus by photodynamic therapy with endogenously generated protoporphyrin IX. Lancet 1996;348: $584-585$

2. van den Boogert J, Houtsmuller AB, de Rooij FW, de Bruin RW, Siersema PD, van Hillegersberg R. Kinetics, localization, and mechanism of 5 -aminolevulinic acid-induced porphyrin accumulation in normal and Barrett's-like rat esophagus. Lasers Surg Med 1999;24:3-13.

3. Ackroyd R, Brown N, Vernon D, Roberts D, Stephenson T, Marcus S, Stoddard C, Reed M. 5-Aminolevulinic acid photosensitization of dysplastic Barrett's esophagus: A pharmacokinetic study. Photochem Photobiol 1999;70:656-662.
4. de Bruijn HS, van der Veen N, Robinson DJ, Star WM. Improvement of 5-aminolevulinic acid based photodynamic therapy in vivo using light fractionation with 75 minute interval. Cancer Res 1999;59:901-904.

5. Robinson DJ, de Bruijn HS, van der Veen N, Stringer MR, Brown SB, Star WM. Fluorescence photobleaching of ALAinduced protoporphyrin IX during photodynamic therapy of normal hairless mouse skin: The effect of light dose and irradiance and the resulting biological effect. Photochem Photobiol 1998;67:141-149.

6. Barr H. Barrett's esophagus: Treatment with 5-aminolevulinic acid photodynamic therapy. Gastrointest Endosc Clin N Am 2000;10:421-437.

7. Gossner L, May A, Sroka R, Stolte M, Hahn EG, Ell C. Photodynamic destruction of high grade dysplasia and early carcinoma of the esophagus after the oral administration of 5-aminolevulinic acid. Cancer 1999;86:19211928.

8. Tan WC, Fulljames C, Stone N, Dix AJ, Shepherd N, Roberts DJ, Brown SB, Krasner N, Barr H. Photodynamic therapy using 5-aminolaevulinic acid for oesophageal adenocarcinoma associated with Barrett's metaplasia. J Photochem Photobiol B 1999;53:75-80.

9. Regula J, MacRobert AJ, Gorchein A, Buonaccorsi GA, Thorpe SM, Spencer GM, Hatfield AR, Bown SG. Photosensitisation and photodynamic therapy of oesophageal, duodenal, and colorectal tumours using 5 aminolaevulinic acid induced protoporphyrin IX - a pilot study. Gut 1995;36:6775 .

10. Maier A, Tomaselli F, Gebhard F, Rehak P, Smolle J, SmolleJuttner FM. Palliation of advanced esophageal carcinoma by photodynamic therapy and irradiation. Ann Thorac Surg 2000;69:1006-1009.

11. Ackroyd R, Brown NJ, Davis MF, Stephenson TJ, Marcus SL, Stoddard CJ, Johnson AG, Reed MW. Photodynamic therapy for dysplastic Barrett's esophagus: A prospective, double blind, randomised, placebo controlled trial. Gut 2000;47:612619.

12. van Staveren HJ, Keijzer M, Keesmaat $T$, Jansen $H$, Kirkel WJ, Beek JF, Star WM. Integrating sphere effect in whole-bladder wall photodynamic therapy: III. Fluence multiplication, optical penetration, and light distribution with an eccentric source for human bladder optical properties. Phys Med Biol 1996;41:579-590.

13. Murrer LHP, Marijnissen HPA, Baas P, Star WM. Applicator for light delivery and in situ light dosimetry during endobronchial photodynamic therapy: First measurements in humans. Lasers Med Sci 1997;12:253-259.

14. Murrer LHP, Marijnissen HPA, Star WM. Ex vivo light dosimetry and Monte Carlo simulations for endobronchial photodynamic therapy. Phys Med Biol 1995;40:18071817.

15. van Staveren HJ, Marijnissen HPA, Aalders MCG, Star WM. Construction, quality assurance and calibration of spherical isotropic fibre optic light diffusers. Lasers Med Sci 1995;10: $137-147$.

16. Marijnissen HP, Star WM. Calibration of isotropic light dosimetry probes based on scattering bulbs in clear media. Phys Med Biol 1996;41:1191-1208.

17. Panjehpour M, Overholt BF, Haydek JM. Light sources and delivery devices for photodynamic therapy in the gastrointestinal tract. Gastrointest Endosc Clin N Am 2000;10: $513-532$.

18. Murrer LH, Marijnissen HP, Star WM. Monte Carlo simulations for endobronchial photodynamic therapy: The influence of variations in optical and geometrical properties and of realistic and eccentric light sources. Lasers Surg Med 1998; 22:193-206.

19. Panjehpour M, Overholt BF, DeNovo RC, Sneed RE, Petersen MG. Centering balloon to improve esophageal photodynamic therapy. Lasers Surg Med 1992;12:631-638.

20. Panjehpour M, Overholt BF, DeNovo RC, Petersen MG, Sneed RE. Comparative study between pulsed and continuous wave lasers for Photofrin photodynamic therapy. Lasers Surg Med 1993;13:296-304. 
21. Overholt BF, Panjehpour M, DeNovo RC, Petersen MG. Photodynamic therapy for esophageal cancer using a 180 degrees windowed esophageal balloon. Lasers Surg Med 1994;14:27-33.

22. van den Bergh $\mathrm{H}$. On the evolution of some endoscopic light delivery systems for photodynamic therapy. Endoscopy 1998;30:392-407.

23. Bays R, Wagnieres GA, Robert D, Braichotte DR, Savary J-F, Monnier P, van den Bergh H. Light dosimetry for photodynamic therapy in the esophagus. Lasers Surg Med 1997;20: $290-303$.

24. van den Boogert J, van Staveren HJ, de Bruin RW, Eikelaar JH, Siersema PD, van Hillegersberg R. Photodynamic therapy for esophageal lesions: Selectivity depends on wave- length, power, and light dose. Ann Thorac Surg 1999;68: 1763-1769.

25. Jode de ML. Monte Carlo simulations of the use of isotropic light dosimetry probes to monitor energy fluence in biological tissue. Phys Med Biol 1999;44:3027-3037.

26. Gossner L, May A, Sroka R, Ell C. A new long-range through-the-scope balloon applicator for photodynamic therapy in the esophagus and cardia. Endoscopy 1999;31:370376.

27. Panjehpour M, Overholt BF, Haydek JM, Lee SG. Results of photodynamic therapy for ablation of dysplasia and early cancer in Barrett's esophagus and effect of oral steroids on stricture formation. Am J Gastroenterol 2000;95:21772184. 